\title{
Existence of Solutions of Fractional Differential Equation with $p$-Laplacian Operator at Resonance
}

\author{
Zhigang Hu, Wenbin Liu, and Jiaying Liu \\ Department of Mathematics, China University of Mining and Technology, Xuzhou 221008, China \\ Correspondence should be addressed to Zhigang Hu; xzhzgya@126.com
}

Received 13 December 2013; Revised 20 February 2014; Accepted 23 February 2014; Published 30 March 2014

Academic Editor: D. Baleanu

Copyright (C) 2014 Zhigang Hu et al. This is an open access article distributed under the Creative Commons Attribution License, which permits unrestricted use, distribution, and reproduction in any medium, provided the original work is properly cited.

By using the extension of Mawhin's continuation theorem due to $\mathrm{Ge}$, we consider boundary value problems for fractional $p$ Laplacian equation. A new result on the existence of solutions for the fractional boundary value problem is obtained, which generalizes and enriches some known results to some extent from the literature.

\section{Introduction and Preliminaries}

Recently, fractional differential equations have played an important role in many fields such as physics, electrical circuits, and control theory (see [1-9]). Many scholars have paid more attention to boundary value problems for fractional differential equations (see [10-25]).

By using a fixed point theorem on a cone, Agarwal et al. (see [10]) considered a two-point boundary value problem at nonresonance given by

$$
\begin{gathered}
D_{0^{+}}^{\alpha} x(t)+f\left(t, x(t), D_{0^{+}}^{\mu} x(t)\right)=0, \\
x(0)=x(1)=0,
\end{gathered}
$$

where $1<\alpha<2, \mu>0$ are real numbers, $\alpha-\mu \geq 1$, and $D_{0^{+}}^{\alpha}$ is the Riemann-Liouville fractional derivative.

By using the coincidence degree theory, Bai (see [20]) considered the following $m$-point fractional boundary value problems:

$$
\begin{gathered}
D_{0^{+}}^{\alpha} u(t)=f\left(t, u(t), D_{0^{+}}^{\alpha-1} u(t)\right)+e(t), \quad 0<t<1, \\
\left.I_{0^{+}}^{2-\alpha} u(t)\right|_{t=0}=0, \quad u(1)=\sum_{i=1}^{m-2} \beta_{i} u\left(\eta_{i}\right),
\end{gathered}
$$

where $1<\alpha \leq 2$ is a real number, $\beta_{i} \in \mathbb{R}, \eta_{i} \in(0,1)$ are given constants such that $\sum_{i=1}^{m-2} \beta_{i} \eta_{i}^{m-1}=1$, and $D_{0^{+}}^{\alpha} I_{0^{+}}^{\alpha}$ are the Riemann-Liouville differentiation and integration.
The turbulent flow in a porous medium is a fundamental mechanics problem. For studying this type of problems, Leibenson (see [26]) introduced the $p$-Laplacian equation as follows:

$$
\left(\phi_{p}\left(x^{\prime}(t)\right)\right)^{\prime}=f\left(t, x(t), x^{\prime}(t)\right),
$$

where $\phi_{p}(s)=|s|^{p-2} s, p>1$. Obviously, $\phi_{p}$ is invertible and its inverse operator is $\phi_{q}$, where $q>1$ is a constant such that $1 / p+1 / q=1$

In the past few decades, many important results relative to (3) with certain boundary value conditions have been obtained. We refer the reader to [27-31] and the references cited therein. However, to the best of our knowledge, there are relatively few results on boundary value problems for fractional $p$-Laplacian equations.

Motivated by the work above, in this paper, we investigate the existence of solutions for boundary value problem (BVP for short) of fractional $p$-Laplacian equation with the following form:

$$
\begin{gathered}
D_{0^{+}}^{\beta} \phi_{p}\left(D_{0^{+}}^{\alpha} x(t)\right)=f\left(t, x(t), D_{0^{+}}^{\alpha} x(t)\right), \quad t \in[0,1], \\
D_{0^{+}}^{\alpha} x(0)=D_{0^{+}}^{\alpha} x(1)=x^{\prime}(0)=0,
\end{gathered}
$$

where $0<\beta \leq 1,1<\alpha \leq 2, D_{0^{+}}^{\alpha}$ is Caputo fractional derivative, and $f:[0,1] \times \mathbb{R}^{2} \rightarrow \mathbb{R}$ is continuous. 
BVP (4) happens to be at resonance in the sense that its associated linear homogeneous boundary value problem

$$
\begin{gathered}
D_{0^{+}}^{\beta} \phi_{p}\left(D_{0^{+}}^{\alpha} x(t)\right)=0, \quad t \in[0,1], \\
D_{0^{+}}^{\alpha} x(0)=D_{0^{+}}^{\alpha} x(1)=x^{\prime}(0)=0
\end{gathered}
$$

has a nontrivial solution $x(t)=c$, where $c \in \mathbb{R}$.

For the convenience of the reader, we present here some necessary basic knowledge and definitions about fractional calculus theory, which can be found, for instance, in [32-35].

Definition 1. The Riemann-Liouville fractional integral operator of order $\alpha>0$ of a function $x:(0,+\infty) \rightarrow \mathbb{R}$ is given by

$$
I_{0^{+}}^{\alpha} x(t)=\frac{1}{\Gamma(\alpha)} \int_{0}^{t}(t-s)^{\alpha-1} x(s) d s,
$$

provided that the right side integral is pointwise defined on $(0,+\infty)$.

Definition 2. The Caputo fractional derivative of order $\alpha>0$ of a continuous function $x:(0,+\infty) \rightarrow \mathbb{R}$ is given by

$$
D_{0^{+}}^{\alpha} x(t)=I_{0^{+}}^{n-\alpha} \frac{d^{n} x(t)}{d t^{n}}=\frac{1}{\Gamma(n-\alpha)} \int_{0}^{t}(t-s)^{n-\alpha-1} x^{(n)}(s) d s,
$$

where $n$ is the smallest integer greater than or equal to $\alpha$, provided that the right side integral is pointwise defined on $(0,+\infty)$.

Lemma 3. Assume that $D_{0^{+}}^{\alpha} x \in C[0,1], \alpha>0$. Then

$$
I_{0^{+}}^{\alpha} D_{0^{+}}^{\alpha} x(t)=x(t)+c_{0}+c_{1} t+c_{2} t^{2}+\cdots+c_{n-1} t^{n-1}
$$

where $c_{i}=-x^{(i)}(0) / i !, i=0,1,2, \ldots, n-1$, and here $n$ is the smallest integer greater than or equal to $\alpha$.

Now, one briefly recalls some notations and an abstract existence result, which can be found in [36].

Definition 4. Let $X$ and $Y$ be two Banach spaces with norms $\|\cdot\|_{X}$ and $\|\cdot\|_{Y}$, respectively. A continuous operator

$$
M: X \cap \operatorname{dom} M \longrightarrow Y
$$

is said to be quasilinear if

(i) $\operatorname{Im} M:=M(X \cap \operatorname{dom} M)$ is a closed subset of $Y$,

(ii) $\operatorname{Ker} M:=\{X \cap \operatorname{dom} M: M u=0\}$ is linearly homeomorphic to $\mathbb{R}^{n}, n<\infty$.

Definition 5. Let $X$ be a real Banach space and let $\widehat{X} \subset X$. The operator $P: X \rightarrow \widehat{X}$ is said to be a projector provided that $P^{2}=P, P\left(\lambda_{1} x_{1}+\lambda_{2} x_{2}\right)=\lambda_{1} P\left(x_{1}\right)+\lambda_{2} P\left(x_{2}\right)$ for $x_{1}, x_{2} \in X$ and $\lambda_{1}, \lambda_{2} \in \mathbb{R}$. The operator $Q: X \rightarrow \widehat{X}$ is said to be a semiprojector provided $Q^{2}=Q$.
Definition 6 (see [36]). Let $\widehat{X}=\operatorname{Ker} M$ and let $\widetilde{X}$ be the complement space of $\widehat{X}$ in $X$, and then $X=\widehat{X} \oplus \widetilde{X}$. On the other hand, suppose that $\widehat{Y}$ is a subspace of $Y$ and $\tilde{Y}$ is the complement space of $\widehat{Y}$ in $Y$ so that $Y=\widehat{Y} \oplus \tilde{Y}$. Let $P: X \rightarrow \widehat{X}$ be a projector, let $Q: Y \rightarrow \widehat{Y}$ be a semiprojector, and let $\Omega \subset X$ be an open and bounded set with origin $\theta \in \Omega$, where $\theta$ is the origin of a linear space.

Suppose that $N_{\lambda}: \bar{\Omega} \rightarrow Y, \lambda \in[0,1]$, is a continuous operator. Denote $N_{1}$ by $N$. Let $\Sigma_{\lambda}=\left\{x \in \bar{\Omega}: M x=N_{\lambda} x\right\}$. $N_{\lambda}$ is said to be $M$-compact in $\bar{\Omega}$ if there is $\widehat{Y} \subset Y$ with $\operatorname{dim} \widehat{Y}$ $=\operatorname{dim} \widehat{X}$ and an operator $R: \bar{\Omega} \times[0,1] \rightarrow X$ continuous and compact such that, for $\lambda \in[0,1]$,

$$
\begin{gathered}
(I-Q) N_{\lambda}(\bar{\Omega}) \subset \operatorname{Im} M \subset(I-Q) Y, \\
Q N_{\lambda} x=\theta, \quad \lambda \in(0,1) \Longleftrightarrow Q N x=\theta,
\end{gathered}
$$

$R(\cdot, 0)$ is the zero operator and $\left.R(\cdot, \lambda)\right|_{\Sigma_{\lambda}}=\left.(I-P)\right|_{\Sigma_{\lambda}}$,

$$
M[P+R(\cdot, \lambda)]=(I-Q) N_{\lambda} .
$$

Lemma 7 (see [36] Ge-Mawhin's continuation theorem). Let $X$ and $Y$ be two Banach spaces with norms $\|\cdot\|_{X}$ and $\|\cdot\|_{Y}$, respectively. $\Omega \subset X$ is an open and bounded nonempty set. Suppose that

$$
M: X \cap \operatorname{dom} M \longrightarrow Y
$$

is a quasilinear operator and

$$
N_{\lambda}: \bar{\Omega} \longrightarrow Y, \quad \lambda \in[0,1]
$$

is $M$-compact in $\bar{\Omega}$. In addition, if

$\left(\mathrm{C}_{1}\right) M x \neq N_{\lambda} x, \forall(x, \lambda) \in(\operatorname{dom} M \cap \partial \Omega) \times(0,1)$,

$\left(\mathrm{C}_{2}\right) \mathrm{Q} N x \neq 0$, for $x \in \operatorname{dom} M \cap \partial \Omega$,

$\left(\mathrm{C}_{3}\right) \operatorname{deg}(J Q N, \operatorname{Ker} M \cap \Omega, 0) \neq 0$,

where $N=N_{1}$, then the equation $M x=N x$ has at least one solution in $\bar{\Omega}$.

In this paper, we take $Y=C[0,1]$ with the norm $\|x\|_{\infty}=$ $\max _{t \in[0,1]}|x(t)|$ and $X=\left\{x \mid x, D_{0^{+}}^{\alpha} x \in Y\right\}$ with the norm $\|x\|_{X}=\max \left\{\|x\|_{\infty},\left\|D_{0^{+}}^{\alpha} x\right\|_{\infty}\right\}$. By means of the linear functional analysis theory, we can prove that $X$ is a Banach space.

Define the operator $M: \operatorname{dom} M \subset X \rightarrow Y$ by

$$
M x=D_{0^{+}}^{\beta} \phi_{p}\left(D_{0^{+}}^{\alpha} x\right) \text {, }
$$

where

$$
\begin{aligned}
& \operatorname{dom} M=\left\{x \in X \mid D_{0^{+}}^{\beta} \phi_{p}\left(D_{0^{+}}^{\alpha} x\right) \in Y,\right. \\
&\left.D_{0^{+}}^{\alpha} x(0)=D_{0^{+}}^{\alpha} x(1)=x^{\prime}(0)=0\right\} .
\end{aligned}
$$

Define the operator $N: X \rightarrow Y$ by

$$
N x(t)=f\left(t, x(t), D_{0^{+}}^{\alpha} x(t)\right), \quad \forall t \in[0,1] .
$$

Then BVP (4) is equivalent to the operator equation. Consider

$$
M x=N x, \quad x \in \operatorname{dom} M .
$$




\section{Main Result}

We will always assume that the nonlinearity $f(t, u, v)$ will be retained:

$\left(\mathrm{H}_{1}\right)$ there exist nonnegative functions $a, b, c \in Y$ such that

$$
\begin{array}{rr}
|f(t, u, v)| \leq a(t)+b(t)|u|^{p-1}+c(t)|v|^{p-1}, & \forall t \in[0,1] \\
& (u, v) \in \mathbb{R}^{2}
\end{array}
$$

$\left(\mathrm{H}_{2}\right)$ there exists a constant $B>0$ such that

either

$$
u f(t, u, v)>0, \quad \forall t \in[0,1], v \in \mathbb{R},|u|>B
$$

or

$$
u f(t, u, v)<0, \quad \forall t \in[0,1], v \in \mathbb{R},|u|>B .
$$

Moreover, we will always assume that $f:[0,1] \times \mathbb{R}^{2} \rightarrow \mathbb{R}$ is continuous and

$$
\frac{1}{\Gamma(\beta+1)}\left(\frac{2\|b\|_{\infty}}{(\Gamma(\alpha+1))^{p-1}}+\|c\|_{\infty}\right)<1 .
$$

Now, we begin with some lemmas below.

Lemma 8. Let $M$ be defined by (16), and then

$$
\begin{aligned}
& \text { Ker } M=\{x \in X \mid x(t)=c \in \mathbb{R}, \forall t \in[0,1]\}, \\
& \operatorname{Im} M=\left\{y \in Y \mid \int_{0}^{1}(1-s)^{\beta-1} y(s) d s=0\right\},
\end{aligned}
$$

and $M$ is a quasilinear operator

Proof. By Lemma 3, $D_{0^{+}}^{\beta} \phi_{p}\left(D_{0^{+}}^{\alpha} x(t)\right)=0$ has solution:

$$
\begin{aligned}
x(t) & =c_{0}+c_{1} t+I_{0^{+}}^{\alpha} \phi_{q}\left(c_{2}\right) \\
& =c_{0}+c_{1} t+\frac{\phi_{q}\left(c_{2}\right)}{\Gamma(\alpha+1)} t^{\alpha}, \quad c_{0}, c_{1}, c_{2} \in \mathbb{R},
\end{aligned}
$$

which satisfies

$$
D_{0^{+}}^{\alpha} x(t)=\phi_{q}\left(c_{2}\right)
$$

Combining with the boundary value condition $D_{0^{+}}^{\alpha} x(0)=0$ and $x^{\prime}(0)=0$, we can get that (24) holds.

If $y \in \operatorname{Im} M$, then there exists a function $x \in \operatorname{dom} M$ such that $y(t)=D_{0^{+}}^{\beta} \phi_{p}\left(D_{0^{+}}^{\alpha} x(t)\right)$. Based on Lemma 3, we have

$$
\begin{aligned}
D_{0^{+}}^{\alpha} x(t) & =\phi_{q}\left(I_{0^{+}}^{\beta} y(t)+c\right) \\
& =\phi_{q}\left(\frac{1}{\Gamma(\beta)} \int_{0}^{t}(t-s)^{\beta-1} y(s) d s+c\right), \quad c \in \mathbb{R} .
\end{aligned}
$$

From condition $D_{0^{+}}^{\alpha} x(0)=0$, one has $c=0$. By the condition $D_{0^{+}}^{\alpha} x(1)=0$, we obtain that

$$
\int_{0}^{1}(1-s)^{\beta-1} y(s) d s=0
$$

Thus, we get (25).

Then we have $\operatorname{dim} \operatorname{Ker} M=1$ and $M(\operatorname{dom} M \cap X) \subset Y$ closed. Therefore, $M$ is a quasilinear operator.

Lemma 9. Let $\Omega \subset X$ be an open and bounded set; then $N_{\lambda}$ is $M$-compact in $\bar{\Omega}$.

Proof. Define the continuous projector $P: X \rightarrow \widehat{X}$ and the semiprojector $Q: Y \rightarrow \widehat{Y}$ :

$$
P x(t)=x(0), \quad \forall t \in[0,1],
$$

$$
Q y(t)=\beta \int_{0}^{1}(1-s)^{\beta-1} y(s) d s, \quad \forall t \in[0,1] \text {. }
$$

where $\widehat{X}=\operatorname{Ker} M$ and $\widehat{Y}=\operatorname{Im} Q$.

Obviously, Im $P=\operatorname{Ker} M$ and $P^{2} x(t)=P x(t)$. It follows from $x=(x-P x)+P x$ that $X=\operatorname{Ker} P+\operatorname{Ker} M$. By a simple calculation, we can get $\operatorname{Ker} M \cap \operatorname{Ker} P=\{0\}$. Then we get

$$
X=\operatorname{Ker} M \oplus \operatorname{Ker} P=\widehat{X} \oplus \widetilde{X} \text {. }
$$

By the definition of $Q$, we can get

$$
Q^{2} y=Q y \cdot \beta \int_{0}^{1}(1-s)^{\beta-1} d s=Q y .
$$

Let $y=(y-Q y)+Q y$, where $y-Q y \in \operatorname{Ker} Q=\operatorname{Im} M$, $Q y \in \operatorname{Im} Q$. It follows from $\operatorname{Ker} Q=\operatorname{Im} M$ and $Q^{2} y=Q y$ that $\operatorname{Im} Q \cap \operatorname{Im} M=\{0\}$. Then, we have

$$
Y=\operatorname{Im} Q \oplus \operatorname{Im} M=\widehat{Y} \oplus \tilde{Y} .
$$

Thus

$$
\operatorname{dim} \widehat{X}=\operatorname{dim} \operatorname{Ker} M=\operatorname{dim} \operatorname{Im} Q=\operatorname{dim} \widehat{Y} .
$$

Let $\Omega \subset X$ be an open and bounded set with $\theta \in \Omega$. For each $x \in \Omega$, we can get $Q\left[(I-Q) N_{\lambda} x\right]=0$. Thus, $(I-Q) N_{\lambda} x \in$ $\operatorname{Im} M=\operatorname{Ker} Q$. Take any $y \in \operatorname{Im} M$ in the type $y=(y-Q y)+$ $Q y$. Since $Q y=0$, we can get $(I-Q) y \in Y$. So (10) holds. It is easy to verify (11).

Furthermore, define $R: \bar{\Omega} \times[0,1] \rightarrow \widetilde{X}$ by

$$
\begin{aligned}
& R(x, \lambda)(t) \\
& =\frac{1}{\Gamma(\alpha)} \int_{0}^{t}(t-s)^{\alpha-1} \phi_{q} \\
& \quad \times\left(\frac{1}{\Gamma(\beta)} \int_{0}^{s}(s-\tau)^{\beta-1}\right. \\
& \left.\quad \times\left((I-Q) N_{\lambda} x(\tau)\right) d \tau\right) d s
\end{aligned}
$$


By the continuity of $f$, it is easy to get that $R(x, \lambda)$ is continuous on $\bar{\Omega} \times[0,1]$. Moreover, for all $x \in \bar{\Omega}$, there exists a constant $T>0$ such that $\left.\mid I_{0^{+}}^{\beta}(I-Q) N_{\lambda} x(\tau)\right) \mid \leq T$, so we can easily obtain that $R(\bar{\Omega}, \lambda)$ is uniformly bounded. By the Arzelà-Ascoli theorem, we just need to prove that $R: \bar{\Omega} \times[0,1] \rightarrow \widetilde{X}$ is equicontinuous. Furthermore, for $0 \leq t_{1}<t_{2} \leq 1,(x, \lambda) \in \bar{\Omega} \times[0,1]$, we have

$$
\begin{aligned}
& \left|R(x, \lambda)\left(t_{2}\right)-R(x, \lambda)\left(t_{1}\right)\right| \\
& =\mid I_{0^{+}}^{\alpha} \phi_{q}\left(I_{0^{+}}^{\beta}(I-Q) N_{\lambda} x\left(t_{2}\right)\right) \\
& \quad-I_{0^{+}}^{\alpha} \phi_{q}\left(I_{0^{+}}^{\beta}(I-Q) N_{\lambda} x\left(t_{1}\right)\right) \mid .
\end{aligned}
$$

By $\left|I_{0^{+}}^{\beta}(I-Q) N_{\lambda} x\right| \leq T$, we have

$$
\begin{aligned}
& \left|I_{0^{+}}^{\alpha} \phi_{q}\left(I_{0^{+}}^{\beta}(I-Q) N_{\lambda} x\left(t_{2}\right)\right)-I_{0^{+}}^{\alpha} \phi_{q}\left(I_{0^{+}}^{\beta}(I-Q) N_{\lambda} x\left(t_{1}\right)\right)\right| \\
& \leq \frac{1}{\Gamma(\alpha)} \mid \int_{0}^{t_{2}}\left(t_{2}-s\right)^{\alpha-1} \phi_{q}\left(I_{0^{+}}^{\beta}(I-Q) N_{\lambda} x(s)\right) d s \\
& \quad-\int_{0}^{t_{1}}\left(t_{1}-s\right)^{\alpha-1} \phi_{q}\left(I_{0^{+}}^{\beta}(I-Q) N_{\lambda} x(s)\right) d s \mid \\
& \leq \frac{\phi_{q}(T)}{\Gamma(\alpha)}\left[\int_{0}^{t_{1}}\left(t_{2}-s\right)^{\alpha-1}-\left(t_{1}-s\right)^{\alpha-1} d s\right. \\
& \left.\quad+\int_{t_{1}}^{t_{2}}\left(t_{2}-s\right)^{\alpha-1} d s\right] \\
& =\frac{\phi_{q}(T)}{\Gamma(\alpha+1)}\left(t_{2}^{\alpha}-t_{1}^{\alpha}\right) .
\end{aligned}
$$

Since $t^{\alpha}$ is uniformly continuous on $[0,1]$, so $R(\bar{\Omega}, \lambda)$ is equicontinuous. Similarly, we can get that $I_{0^{+}}^{\beta}((I-$ Q) $\left.N_{\lambda} x(\tau)\right) \subset C[0,1]$ is equicontinuous, and considering that $\phi_{q}(s)$ is uniformly continuous on $[-T, T]$, we get that $D_{0^{+}}^{\alpha} R(\bar{\Omega}, \lambda)=I_{0^{+}}^{\beta}\left((I-Q) N_{\lambda}(\bar{\Omega})\right)$ is also equicontinuous. So we can obtain that $R(\bar{\Omega}, \lambda) \rightarrow \widetilde{X}$ is compact.

For each $x \in \Sigma_{\lambda}=\left\{x \in \bar{\Omega}: M x=N_{\lambda} x\right\}$, we have $D_{0^{+}}^{\beta} \phi_{p}\left(D_{0^{+}}^{\alpha} x(t)\right)=N_{\lambda} x(t) \in \operatorname{Im} M$. Thus,

$$
\begin{aligned}
& R(x, \lambda)(t) \\
& =\frac{1}{\Gamma(\alpha)} \int_{0}^{t}(t-s)^{\alpha-1} \phi_{q} \\
& \quad \times\left(\frac{1}{\Gamma(\beta)} \int_{0}^{s}(s-\tau)^{\beta-1}\right. \\
& \left.\quad \times\left((I-Q) N_{\lambda} x(\tau)\right) d \tau\right) d s .
\end{aligned}
$$

$$
\begin{aligned}
&=\frac{1}{\Gamma(\alpha)} \int_{0}^{t}(t-s)^{\alpha-1} \phi_{q} \\
& \times\left(\frac{1}{\Gamma(\beta)} \int_{0}^{s}(s-\tau)^{\beta-1}\right. \\
&\left.\quad \times D_{0^{+}}^{\beta} \phi_{p}\left(D_{0^{+}}^{\alpha} x(\tau)\right) d \tau\right) d s,
\end{aligned}
$$

which together with $D_{0^{+}}^{\alpha} x(0)=x^{\prime}(0)=0$ yields that

$$
R(x, \lambda)(t)=x(t)-x(0)=[(I-P) x](t) .
$$

It is easy to verify that $R(x, 0)(t)$ is the zero operator. So (12) holds.

On the other hand, consider

$$
\begin{aligned}
& M[P x+R(x, \lambda)](t) \\
& =M\left[\frac{1}{\Gamma(\alpha)}\right. \\
& \times \int_{0}^{t}(t-s)^{\alpha-1} \phi_{q}\left(\frac{1}{\Gamma(\beta)} \int_{0}^{s}(s-\tau)^{\beta-1}\right. \\
& \left.\times\left((I-Q) N_{\lambda} x(\tau)\right) d \tau\right) d s \\
& +x(0)] \\
& =\left[\left((I-Q) N_{\lambda}\right) x\right](t) \text {. }
\end{aligned}
$$

So (13) holds. Then we get that $N_{\lambda}$ is $M$-compact in $\bar{\Omega}$. The proof is complete.

Lemma 10. Suppose that $\left(H_{1}\right),\left(H_{2}\right)$ hold; then the set

$$
\Omega_{1}=\{x \in \operatorname{dom} M \backslash \operatorname{Ker} M \mid M x=\lambda N x, \lambda \in(0,1)\}
$$
is bounded.

Proof. Take $x \in \Omega_{1}$; then $M x=\lambda N x, D_{0^{+}}^{\alpha} x(0)=x^{\prime}(0)=0$, and $N x \in \operatorname{Im} M$. By (25), we have

$$
\int_{0}^{1}(1-s)^{\beta-1} f\left(s, x(s), D_{0^{+}}^{\alpha} x(s)\right) d s=0 .
$$

Then, by the integral mean value theorem, there exists a constant $\xi \in(0,1)$ such that $f\left(\xi, x(\xi), D_{0^{+}}^{\alpha} x(\xi)\right)=0$. So, from $\left(H_{2}\right)$, we get $|x(\xi)| \leq B$. By $x^{\prime}(0)=0$, we get

$$
\begin{aligned}
x(t) & =x(0)+x^{\prime}(0) t+I_{0^{+}}^{\alpha} D_{0^{+}}^{\alpha} x(t) \\
& =x(0)+\frac{1}{\Gamma(\alpha)} \int_{0}^{t}(t-s)^{\alpha-1} D_{0^{+}}^{\alpha} x(s) d s .
\end{aligned}
$$

Take $t=\xi$; we have

$$
x(\xi)=x(0)+\frac{1}{\Gamma(\alpha)} \int_{0}^{\xi}(\xi-s)^{\alpha-1} D_{0^{+}}^{\alpha} x(s) d s .
$$


Then we have

$$
\begin{aligned}
|x(0)| & \leq|x(\xi)|+\frac{1}{\Gamma(\alpha)} \int_{0}^{\xi}(\xi-s)^{\alpha-1}\left|D_{0^{+}}^{\alpha} x(s)\right| d s \\
& \leq|x(\xi)|+\frac{1}{\Gamma(\alpha)}\left\|D_{0^{+}}^{\alpha} x\right\|_{\infty} \cdot \frac{1}{\alpha} \xi^{\alpha} \\
& \leq B+\frac{1}{\Gamma(\alpha+1)}\left\|D_{0^{+}}^{\alpha} x\right\|_{\infty} .
\end{aligned}
$$

So we get

$$
\begin{aligned}
|x(t)| & \leq|x(0)|+\frac{1}{\Gamma(\alpha)} \int_{0}^{t}(t-s)^{\alpha-1}\left|D_{0^{+}}^{\alpha} x(s)\right| d s \\
& \leq|x(0)|+\frac{1}{\Gamma(\alpha)}\left\|D_{0^{+}}^{\alpha} x\right\|_{\infty} \cdot \frac{1}{\alpha} t^{\alpha} \\
& \leq B+\frac{2}{\Gamma(\alpha+1)}\left\|D_{0^{+}}^{\alpha} x\right\|_{\infty}, \quad \forall t \in[0,1] .
\end{aligned}
$$

That is,

$$
\|x\|_{\infty} \leq B+\frac{2}{\Gamma(\alpha+1)}\left\|D_{0^{+}}^{\alpha} x\right\|_{\infty}
$$

By $M x=\lambda N x$ and $D_{0^{+}}^{\alpha} x(0)=0$, we get

$$
\begin{aligned}
\phi_{p}\left(D_{0^{+}}^{\alpha} x(t)\right) & =\lambda I_{0^{+}}^{\beta} N x(t) \\
& =\frac{\lambda}{\Gamma(\beta)} \int_{0}^{t}(t-s)^{\beta-1} f\left(s, x(s), D_{0^{+}}^{\alpha} x(s)\right) d s .
\end{aligned}
$$

So, from $\left(H_{1}\right)$, we have

$$
\begin{aligned}
\left|\phi_{p}\left(D_{0^{+}}^{\alpha} x(t)\right)\right| & \leq \frac{1}{\Gamma(\beta)} \int_{0}^{t}(t-s)^{\beta-1}\left|f\left(s, x(s), D_{0^{+}}^{\alpha} x(s)\right)\right| d s \\
\leq & \frac{1}{\Gamma(\beta)} \int_{0}^{t}(t-s)^{\beta-1}\left(a(s)+b(s)|x(s)|^{p-1}\right. \\
& \left.+c(s)\left|D_{0^{+}}^{\alpha} x(s)\right|^{p-1}\right) d s \\
\leq & \frac{1}{\Gamma(\beta)}\left(\|a\|_{\infty}+\|b\|_{\infty}\|x\|_{\infty}^{p-1}\right. \\
& \left.+\|c\|_{\infty}\left\|D_{0^{+}}^{\alpha} x\right\|_{\infty}^{p-1}\right) \cdot \frac{1}{\beta} t^{\beta} \\
& \frac{1}{\Gamma(\beta+1)}\left(\|a\|_{\infty}+\|b\|_{\infty}\|x\|_{\infty}^{p-1}\right.
\end{aligned}
$$

which together with $\left|\phi_{p}\left(D_{0^{+}}^{\alpha} x(t)\right)\right|=\left|D_{0^{+}}^{\alpha} x(t)\right|^{p-1}$ and (47) yields that

$$
\begin{aligned}
\left\|D_{0^{+}}^{\alpha} x\right\|_{\infty}^{p-1} \leq \frac{1}{\Gamma(\beta+1)}[ & \|a\|_{\infty}+\|b\|_{\infty} \\
& \times\left(B+\frac{2}{\Gamma(\alpha+1)}\left\|D_{0^{+}}^{\alpha} x\right\|_{\infty}\right)^{p-1} \\
& \left.+\|c\|_{\infty}\left\|D_{0^{+}}^{\alpha} x\right\|_{\infty}^{p-1}\right] .
\end{aligned}
$$

In view of (23), we can see that there exists a constant $M_{1}>0$ such that

$$
\left\|D_{0^{+}}^{\alpha} x\right\|_{\infty} \leq M_{1}
$$

Thus, from (47), we get

$$
\|x\|_{\infty} \leq B+\frac{2 M_{1}}{\Gamma(\alpha+1)}:=M_{2} .
$$

Combining (51) with (52), we have

$$
\|x\|_{X}=\max \left\{\|x\|_{\infty},\left\|D_{0^{+}}^{\alpha} x\right\|_{\infty}\right\} \leq \max \left\{M_{1}, M_{2}\right\}:=M \text {. }
$$

Therefore, $\Omega_{1}$ is bounded. The proof is complete.

Lemma 11. Suppose that $\left(\mathrm{H}_{2}\right)$ holds; then the set

$$
\Omega_{2}=\{x \in \operatorname{Ker} M \mid N x \in \operatorname{Im} M\}
$$

is bounded.

Proof. For $x \in \Omega_{2}$, we have $x(t)=c, c \in \mathbb{R}$ and $N x \in \operatorname{Im} M$. Then we get

$$
\int_{0}^{1}(1-s)^{\beta-1} f(s, c, 0) d s=0,
$$

which together with $\left(\mathrm{H}_{2}\right)$ implies $|c| \leq B$. Thus, we have

$$
\|x\|_{X} \leq \max \{B, 0\}=B .
$$

Hence, $\Omega_{2}$ is bounded. The proof is complete.

Lemma 12. Suppose that the first part of $\left(\mathrm{H}_{2}\right)$ holds; then the set

$$
\Omega_{3}=\{x \in \operatorname{Ker} M \mid \lambda x+(1-\lambda) Q N x=0, \lambda \in[0,1]\}
$$
is bounded.

Proof. For $x \in \Omega_{3}$, we have $x(t)=c, c \in \mathbb{R}$, and

$$
\lambda c+(1-\lambda) \beta \int_{0}^{1}(1-s)^{\beta-1} f(s, c, 0) d s=0 .
$$

If $\lambda=0$, then $|c| \leq B$ because of the first part of $\left(\mathrm{H}_{2}\right)$. If $\lambda \in(0,1]$, we can also obtain $|c| \leq B$. Otherwise, if $|c|>B$, in view of the first part of $\left(\mathrm{H}_{2}\right)$, one has

$$
\lambda c^{2}+(1-\lambda) \beta \int_{0}^{1}(1-s)^{\beta-1} c f(s, c, 0) d s>0,
$$

which contradicts (58). Therefore, $\Omega_{3}$ is bounded. The proof is complete. 
Remark 13. If the second part of $\left(\mathrm{H}_{2}\right)$ holds, then the set

$$
\Omega_{3}^{\prime}=\{x \in \operatorname{Ker} M-\lambda x+(1-\lambda) Q N x=0, \lambda \in[0,1]\}
$$

is bounded.

Theorem 14. Let $f:[0,1] \times \mathbb{R}^{2} \rightarrow \mathbb{R}$ be continuous. Suppose that $\left(H_{1}\right),\left(H_{2}\right)$ hold. Then BVP $(4)$ has at least one solution.

Proof. Set $\Omega=\left\{x \in X \mid\|x\|_{X}<\max \{M, B\}+1\right\}$. It follows from Lemmas 8 and 9 that $M$ is a quasilinear operator and $N_{\lambda}$ is $M$-compact on $\bar{\Omega}$. By Lemmas 10 and 11 , we get that the following two conditions are satisfied:

$$
\begin{aligned}
& \left(\mathrm{C}_{1}\right) M x \neq N_{\lambda} x, \forall(x, \lambda) \in(\operatorname{dom} M \cap \partial \Omega) \times(0,1), \\
& \left(\mathrm{C}_{2}\right) Q N x \neq 0, \text { for } x \in \operatorname{dom} M \cap \partial \Omega .
\end{aligned}
$$

Take

$$
H(x, \lambda)= \pm \lambda x+(1-\lambda) Q N x .
$$

According to Lemma 12 (or Remark 13), we know that $H(x, \lambda) \neq 0$ for $x \in \operatorname{Ker} M \cap \partial \Omega$. Therefore

$$
\begin{aligned}
\operatorname{deg}( & \left.\left.Q N\right|_{\operatorname{Ker} M}, \Omega \cap \operatorname{Ker} M, 0\right) \\
& =\operatorname{deg}(H(\cdot, 0), \Omega \cap \operatorname{Ker} M, 0) \\
& =\operatorname{deg}(H(\cdot, 1), \Omega \cap \operatorname{Ker} M, 0) \\
& =\operatorname{deg}( \pm I, \Omega \cap \operatorname{Ker} M, 0) \neq 0 .
\end{aligned}
$$

So the condition $\left(C_{3}\right)$ of Lemma 7 is satisfied. By Lemma 7 , we can get that $M x=N x$ has at least one solution in $\operatorname{dom} M \cap$ $\bar{\Omega}$. Therefore BVP (4) has at least one solution. The proof is complete.

\section{Example}

In this section, we will give an example to illustrate our main result.

Example 1. Consider the following BVP:

$$
\begin{aligned}
& D_{0^{+}}^{3 / 4} \phi_{3}\left(D_{0^{+}}^{3 / 2} x(t)\right)=-\frac{25}{3}+\frac{1}{3} x^{2}(t) \\
&+t e^{-\left|D_{0^{+}}^{3 / 2} x(t)\right|}, \quad t \in[0,1], \\
& D_{0^{+}}^{3 / 2} x(0)=D_{0^{+}}^{3 / 2} x(1)=x^{\prime}(0)=0 .
\end{aligned}
$$

Corresponding to BVP (4), we get that $p=3, \alpha=3 / 2, \beta=$ $3 / 4$, and

$$
f(t, u, v)=-\frac{25}{3}+\frac{1}{3} u^{2}+t e^{-|v|} .
$$

Choose $a(t)=10, b(t)=1 / 3, c(t)=0, B=5$. By a simple calculation, we can get that $\|b\|_{\infty}=1 / 3,\|c\|_{\infty}=0$ and

$$
\frac{1}{\Gamma(3 / 4+1)}\left(\frac{2 / 3}{(\Gamma(3 / 2+1))^{2}}+0\right)<1 .
$$

Obviously, BVP (63) satisfies all conditions of Theorem 14. Hence, it has at least one solution.

\section{Conclusions}

In this paper, the boundary value problem for $p$-Laplacian equation at resonance is investigated. In view of the boundary value problem (4) is equivalent to the operator equation (19); we only need to find a fixed point of the operator equation (19). Firstly, we established the sufficient conditions of existence of boundary value problem for $p$-Laplacian equation. Then, by using the extension of Mawhin's continuation theorem due to Ge, we got the fixed point of operator equation (19).

\section{Conflict of Interests}

The authors declare that there is no conflict of interests regarding the publication of this paper.

\section{Acknowledgments}

This research was supported by the Fundamental Research Funds for the Central Universities (2013XK03) and the National Natural Science Foundation of China (11271364).

\section{References}

[1] R. Metzler and J. Klafter, "Boundary value problems for fractional diffusion equations," Physica A, vol. 278, no. 1-2, pp. 107$125,2000$.

[2] H. Scher and E. W. Montroll, "Anomalous transit-time dispersion in amorphous solids," Physical Review B, vol. 12, no. 6, pp. 2455-2477, 1975.

[3] F. Mainardi, "Fractional diffusive waves in viscoelastic solids," in Nonlinear Waves in Solids, J. L. Wegner and F. R. Norwood, Eds., pp. 93-97, Fairfield, 1995.

[4] K. Diethelm and A. D. Freed, "On the solution of nonlinear fractional order differential equations used in the modeling of viscoplasticity," in Scientific Computing in Chemical Engineering II-Computational Fluid Dynamics, Reaction Engineering and Molecular Properties, F. Keil, W. Mackens, H. Voss, and J. Werther, Eds., pp. 217-224, Springer, Heidelberg, Germany, 1999.

[5] L. Gaul, P. Klein, and S. Kemple, "Damping description involving fractional operators," Mechanical Systems and Signal Processing, vol. 5, no. 2, pp. 81-88, 1991.

[6] W. G. Glockle and T. F. Nonnenmacher, "A fractional calculus approach to self-similar protein dynamics," Biophysical Journal, vol. 68, no. 1, pp. 46-53, 1995.

[7] F. Mainardi, "Fractional calculus: some basic problems in continuum and statistical mechanics," in Fractals and Fractional Calculus in Continuum Mechanics, vol. 378, pp. 291-348, Springer, Vienna, Austria, 1997.

[8] R. Metzler, W. Schick, H.-G. Kilian, and T. F. Nonnenmacher, "Relaxation in filled polymers: a fractional calculus approach," The Journal of Chemical Physics, vol. 103, no. 16, pp. 7180-7186, 1995.

[9] K. B. Oldham and J. Spanier, The Fractional Calculus, Academic Press, NewYork, NY, USA, 1974.

[10] R. P. Agarwal, D. O'Regan, and S. Staněk, "Positive solutions for Dirichlet problems of singular nonlinear fractional differential equations," Journal of Mathematical Analysis and Applications, vol. 371, no. 1, pp. 57-68, 2010. 
[11] Z. Bai and H. Lü, "Positive solutions for boundary value problem of nonlinear fractional differential equation," Journal of Mathematical Analysis and Applications, vol. 311, no. 2, pp. 495-505, 2005.

[12] E. R. Kaufmann and E. Mboumi, "Positive solutions of a boundary value problem for a nonlinear fractional differential equation," Electronic Journal of Qualitative Theory of Differential Equations, vol. 311, no. 2, pp. 495-505, 2008.

[13] H. Jafari and V. Daftardar-Gejji, "Positive solutions of nonlinear fractional boundary value problems using Adomian decomposition method," Applied Mathematics and Computation, vol. 180, no. 2, pp. 700-706, 2006.

[14] M. Benchohra, S. Hamani, and S. K. Ntouyas, "Boundary value problems for differential equations with fractional order and nonlocal conditions," Nonlinear Analysis. Theory, Methods \& Applications, vol. 71, no. 7-8, pp. 2391-2396, 2009.

[15] S. Liang and J. Zhang, "Positive solutions for boundary value problems of nonlinear fractional differential equation," Nonlinear Analysis. Theory, Methods \& Applications, vol. 71, no. 11, pp. 5545-5550, 2009.

[16] S. Zhang, "Positive solutions for boundary-value problems of nonlinear fractional differential equations," Electronic Journal of Differential Equations, vol. 36, pp. 1-12, 2006.

[17] N. Kosmatov, "A boundary value problem of fractional order at resonance," Electronic Journal of Differential Equations, vol. 135, pp. 1-10, 2010.

[18] Z. Wei, W. Dong, and J. Che, "Periodic boundary value problems for fractional differential equations involving a RiemannLiouville fractional derivative," Nonlinear Analysis. Theory, Methods \& Applications, vol. 73, no. 10, pp. 3232-3238, 2010.

[19] Z. Bai and Y. Zhang, "Solvability of fractional three-point boundary value problems with nonlinear growth," Applied Mathematics and Computation, vol. 218, no. 5, pp. 1719-1725, 2011.

[20] Z. Bai, "Solvability for a class of fractional m-point boundary value problem at resonance," Computers \& Mathematics with Applications, vol. 62, no. 3, pp. 1292-1302, 2011.

[21] B. Ahmad and S. Sivasundaram, "On four-point nonlocal boundary value problems of nonlinear integro-differential equations of fractional order," Applied Mathematics and Computation, vol. 217, no. 2, pp. 480-487, 2010.

[22] G. Wang, B. Ahmad, and L. Zhang, "Impulsive anti-periodic boundary value problem for nonlinear differential equations of fractional order," Nonlinear Analysis. Theory, Methods \& Applications, vol. 74, no. 3, pp. 792-804, 2011.

[23] W. Zhong, X. Yang, and F. Gao, "A Cauchy problem for some local fractional abstract differential equation with fractal conditions," Journal of Applied Functional Analysis, vol. 8, no. 1, pp. 92-99, 2013.

[24] Z. Hu and W. Liu, "Solvability for fractional order boundary value problems at resonance," Boundary Value Problems, vol. 20, article 20, 2011.

[25] W. Jiang, "The existence of solutions to boundary value problems of fractional differential equations at resonance," Nonlinear Analysis. Theory, Methods \& Applications, vol. 74, no. 5, pp. 1987-1994, 2011.

[26] L. S. Leibenson, "General problem of the movement of a compressible fluid in a porous medium," Izvestiia Akademii NaukKirgizsko, vol. 9, pp. 7-10, 1945.

[27] T. Chen, W. Liu, and Z. Hu, "A boundary value problem for fractional differential equation with p-Laplacian operator at resonance," Nonlinear Analysis. Theory, Methods \& Applications, vol. 75, no. 6, pp. 3210-3217, 2012.

[28] D. Jiang and W. Gao, "Upper and lower solution method and a singular boundary value problem for the one-dimensional pLaplacian," Journal of Mathematical Analysis and Applications, vol. 252, no. 2, pp. 631-648, 2000.

[29] L. F. Lian and W. G. Ge, “The existence of solutions of m-point pLaplacian boundary value problems at resonance," Acta Mathematicae Applicatae Sinica, vol. 28, no. 2, pp. 288-295, 2005.

[30] B. Liu and J. S. Yu, "Existence of solutions for the periodic boundary value problems with p-Laplacian operator," Journal of Systems Science and Mathematical Sciences, vol. 23, no. 1, pp. 76-85, 2003.

[31] J. J. Zhang, W. B. Liu, J. B. Ni, and T. Y. Chen, "Multiple periodic solutions of p-Laplacian equation with one-side Nagumo condition," Journal of the Korean Mathematical Society, vol. 45, no. 6, pp. 1549-1559, 2008.

[32] X. Yang, Local Fractional Functional Analysis and Its Applications, Asian Academic Publisher Limited, Hong Kong, China, 2011.

[33] I. Podlubny, Fractional Differential Equations, vol. 198, Academic Press, San Diego, Calif, USA, 1999.

[34] S. G. Samko, A. A. Kilbas, and O. I. Marichev, Fractional Integrals and Derivatives (Theory and Applications), Gordon and Breach Science Publishers, Yverdon, Switzerland, 1993.

[35] A. A. Kilbas, H. M. Srivastava, and J. J. Trujillo, Theory and Applications of Fractional Differential Equations, Elsevier Science, Amsterdam, The Netherlands, 2006.

[36] W. Ge and J. Ren, "An extension of Mawhin's continuation theorem and its application to boundary value problems with a pLaplacian," Nonlinear Analysis. Theory, Methods \& Applications, vol. 58, no. 3-4, pp. 477-488, 2004. 


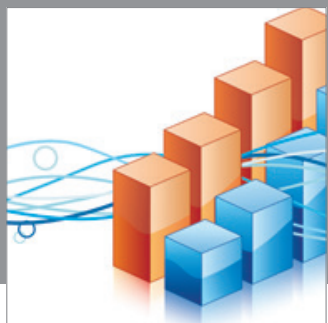

Advances in

Operations Research

mansans

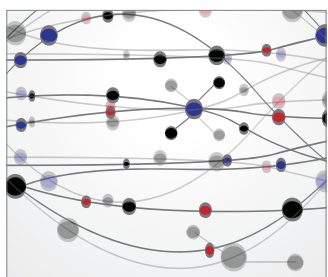

The Scientific World Journal
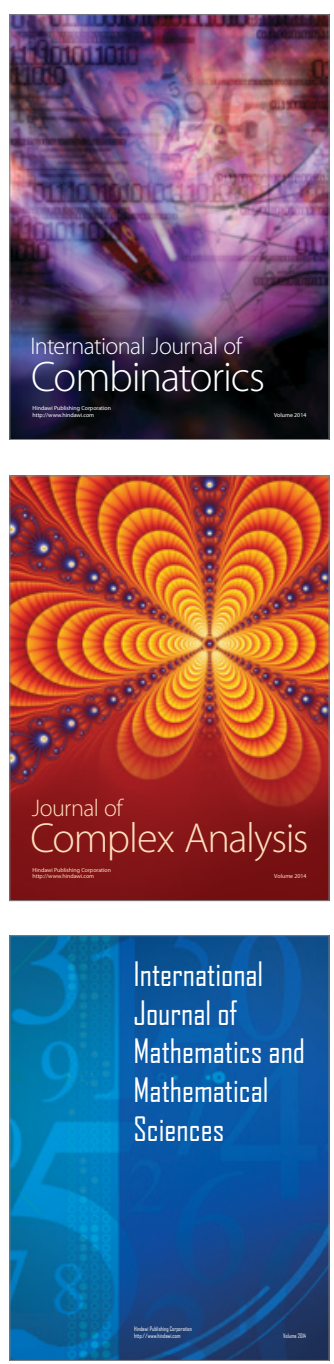
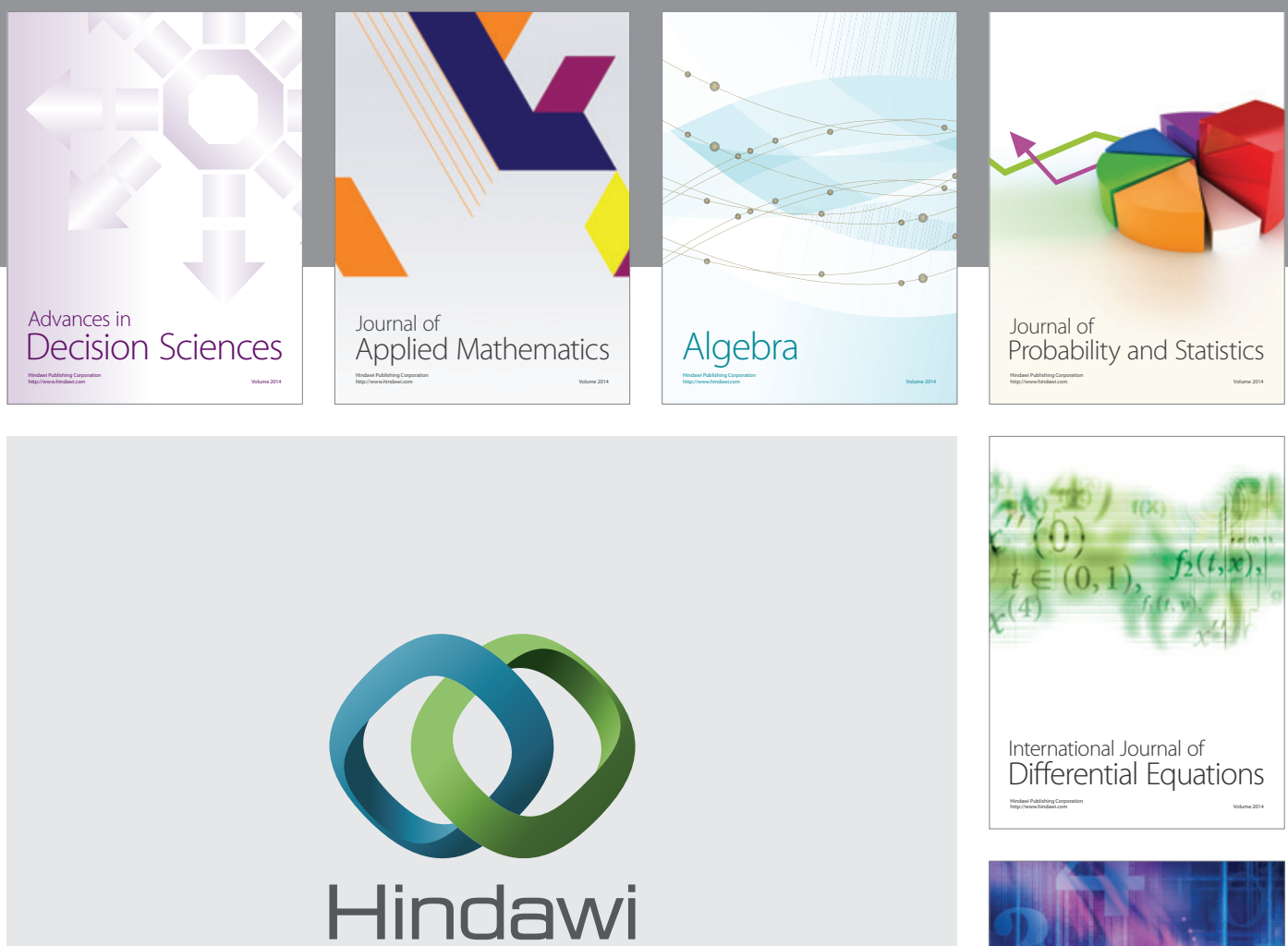

Submit your manuscripts at http://www.hindawi.com
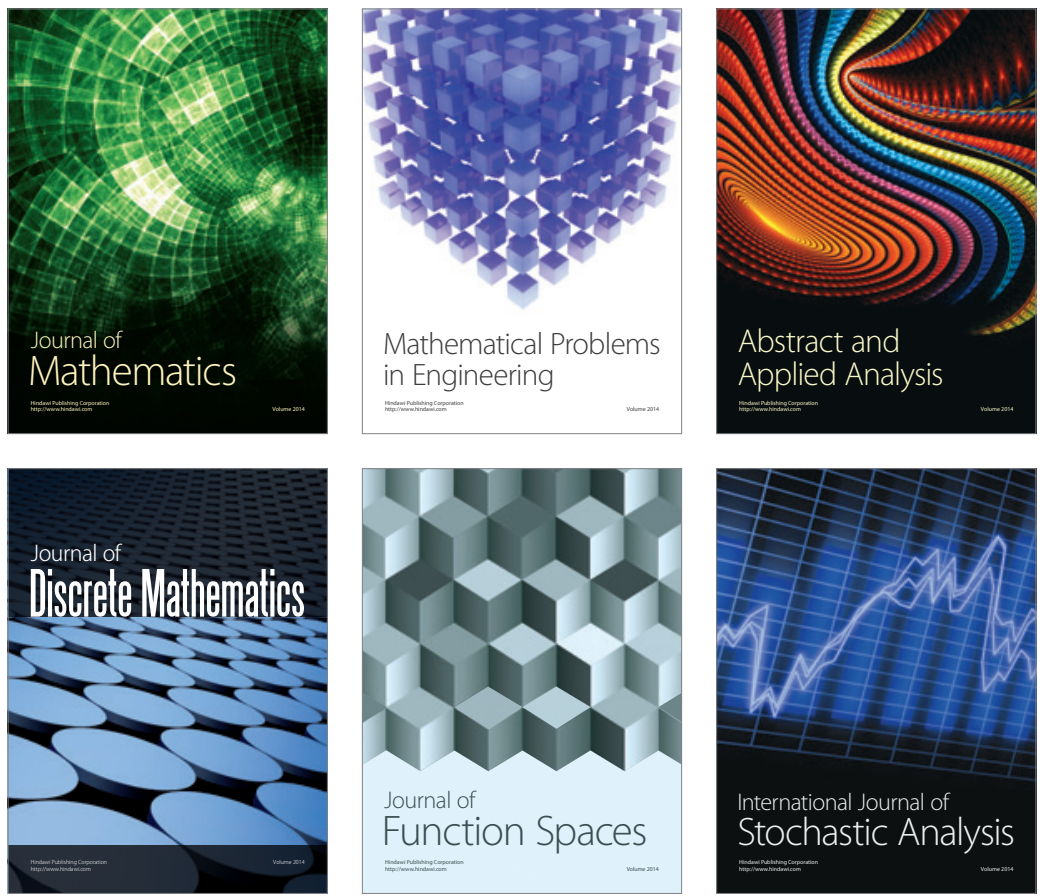

Journal of

Function Spaces

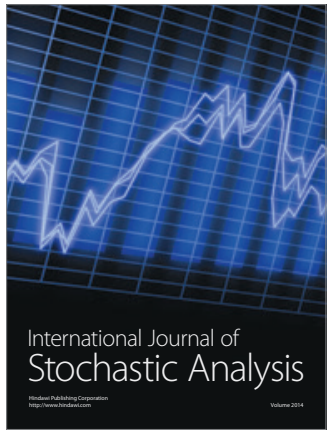

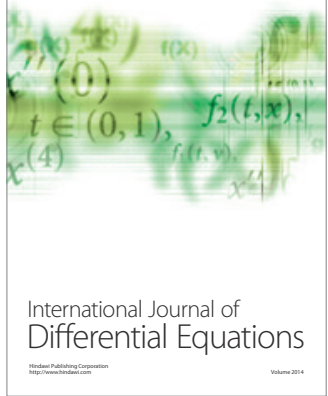
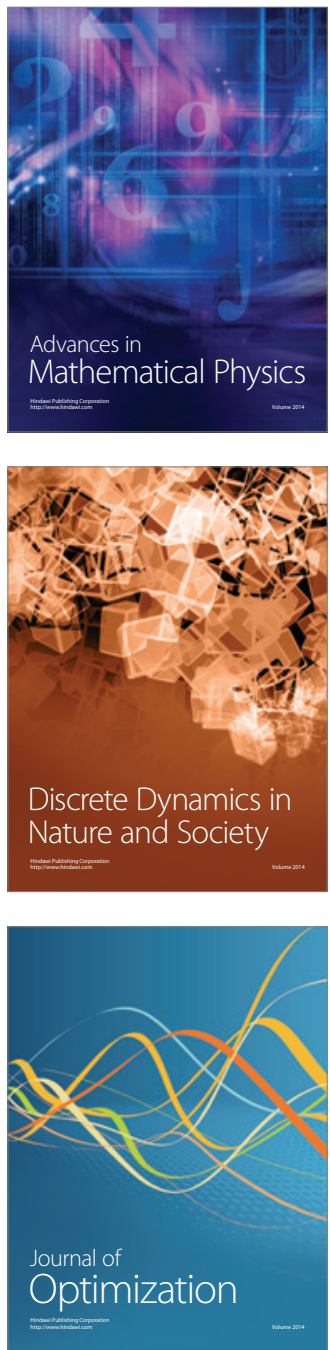